\title{
ChemComm
}

Cite this: Chem. Commun., 2013, 49, 5198

Received 18th March 2013, Accepted 9th April 2013

DOI: $10.1039 / c 3 c c 41932 k$

\section{A cellular logic circuit for the detection of bacterial pore-forming toxins $† \ddagger$}

\author{
Kui Zhu, ${ }^{*}$ Ulaş Acaröz and Erwin Märtlbauer
}

www.rsc.org/chemcomm

We present a cellular logic circuit for deciphering the profiles of toxin production in $B$. cereus, using multiple readout techniques based on the pore formation on the cell membrane. This new assay enables the simultaneous detection of seven biomarkers in pathogenic strains from various samples.

Bacterial toxins are produced by both Gram-positive and Gramnegative pathogens throughout their life cycles to adapt many different niches, which contribute to their virulence or sometimes are essential for their survival. ${ }^{1}$ Some bacterial species are responsible for different illnesses due to the expression of diverse pathogenic factors, as exemplified by Escherichia coli including the notorious $0157: \mathrm{H} 7$ strain. ${ }^{2}$ Although there is a great variety of virulence factors participating in bacterial pathogenesis, pore-forming toxins (PFTs) comprise the largest group ( $>25 \%$ ) of all bacterial protein toxins. ${ }^{3}$ PFTs are generally produced by bacteria in single soluble monomeric forms, alpha-PFTs and beta-PFTs (depending on the mode of membrane binding, either by alpha-helical or beta-sheet units), and can perform highly ordered tasks by harnessing the versatile monomeric proteins to assemble into oligomeric structures. ${ }^{4,5}$ The fact that the self-assembly of oligomers forms a highly ordered ring-like structure to penetrate the target cell membrane, which can be recognized as nanomachines, is particularly fascinating. ${ }^{1,4 a}$

Bacillus cereus (B. cereus) is a Gram-positive, widely distributed opportunistic bacterium, responsible for two types of foodborne disease, the emetic and the diarrheal syndromes, which are caused by different toxins. ${ }^{6}$ The emetic toxin is a heat-stable and ring-formed peptide causing vomiting, while the three protein enterotoxins are the main reasons for diarrhea. All the three toxins, nonhaemolytic enterotoxin (Nhe),

Institute of Food Safety, Faculty of Veterinary Medicine, Ludwig-MaximiliansUniversity Munich, Schönleutnerstr. 8, 85764 Oberschleissheim, Germany. E-mail: zhukcau@gmail.com; Fax: +49 892180 78576; Tel: +49 89218078602 $\dagger$ This paper is dedicated to my dear Prof. Dr Dr h. c. Erwin Märtlbauer for his coming birthday.

‡ Electronic supplementary information (ESI) available: Experimental details and supporting figures and tables. See DOI: 10.1039/c3cc41932k haemolysin $\mathrm{BL}(\mathrm{Hbl})$ and cytotoxin $\mathrm{K}$ (CytK), belong to the group of PFTs. ${ }^{6 a, 7}$ Both Nhe and $\mathrm{Hbl}$ are tripartite toxins and alpha-PFTs, which consist of NheA, B and C, Hbl-L1, L2 and B, respectively. CytK, however, is a beta-PFT and consists of homogeneous monomers. Here, all these toxins were employed as models for both alpha- and beta-PFTs to form pores on the cell membrane for constructing a cellular logic system. And the subsequent cell events can be monitored by determining the release of lactate dehydrogenase ( $\mathrm{LDH}$ assay), activity of mitochondrion (WST assay) and propidium iodide binding to DNA (PI assay), as shown in Scheme 1a.

Biocomputing systems with sophisticated information processing possess various applications. ${ }^{8}$ Novel biosensors, based on Boolean logic operations with a simply binary Yes/No answer, have been achieved in abiotic biocomputing systems based on proteins or nucleotides, holding a glorious prospect for bioanalytical applications. ${ }^{9}$ Bacterial cell based logic gates

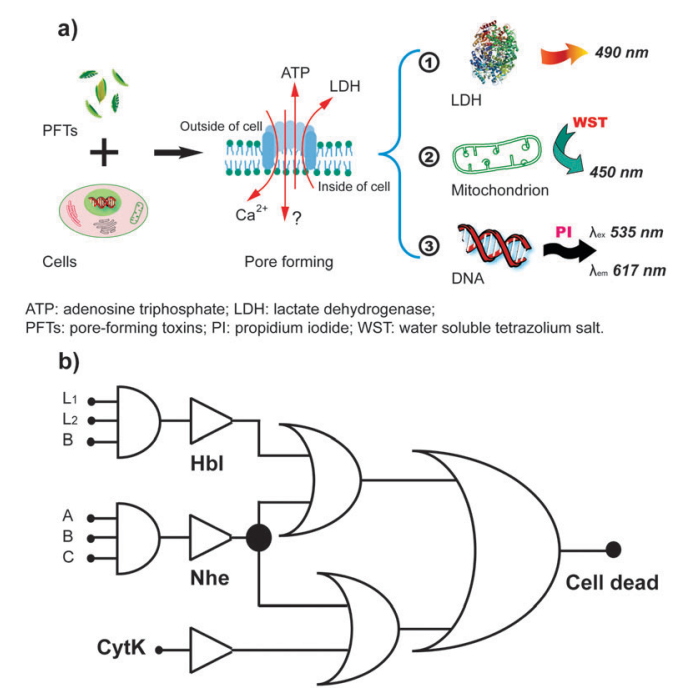

Scheme 1 Cellular logic circuit for the detection of pore-forming toxins (PFTs) from $B$. cereus. (a) Schematic representation of 3 assays for evaluating the viability of the cells treated with PFTs. (b) Equivalent circuit of the Nhe, $\mathrm{Hbl}$ and CytK based cellular circuit. 
have also been developed for different aims. ${ }^{10}$ However, the use of mammalian cells to perform biocomputing operations has been rarely reported. ${ }^{11}$ Compared with individual logic gates, logic circuits composed of the concatenated logic gates performing complex operations are urgently needed for the challenging analysis of multiple inputs with limited selectivity. For example, this new approach can provide an alternative for simultaneous analysis of the presence of multiple biomarkers at different concentration levels, e.g., when a single biomarker analysis is not enough to draw a convincing conclusion. ${ }^{12}$ Herein, we propose a sophisticated logic circuit that included several types of concatenated cellular logic gates to perform Boolean logic operations processing multiple input signals for simultaneously computing the presence of PFTs in B. cereus, as shown in Scheme 1b. In this work, the presence of all seven proteinic biomarkers of three PFTs in B. cereus is reported using African green monkey kidney epithelial (Vero) cells, to generate a qualitative binary result in the Yes/No form.

As shown in Scheme 1b, the logic circuit is composed of several gates based on the three PFTs, which covers different toxin-types of $B$. cereus. Some strains can produce individual CytK or Nhe toxins, and the expression of $\mathrm{Hbl}$ proteins is always coupled with Nhe production. No natural strain can solely express $\mathrm{Hbl}$ without Nhe. The combination of CytK, Nhe and $\mathrm{Hbl}$ sometimes happens in a single strain, however, no strain harboring only CytK and $\mathrm{Hbl}$ has been identified yet. Any one of the complete toxins can trigger cytotoxicity to Vero cells, e.g., the flat cells could be released from the adherent surface and suspend in the cell culture medium with round morphology when they were treated with the tripartite Nhe complex (Fig. S1, ESI $\neq$ ). Since none of the three components in Nhe is enough for triggering full cytotoxicity to Vero cells, only simultaneous existence of all three components would provide potential toxicity. So, NheA, B and C are utilized as multiple inputs, the presence and absence are defined as 'True' input or ' 1 ', and 'False' input or ' 0 ', respectively. The viability of cells is employed as output. To further quantify the information of output signals, the concept of a cytotoxic titer is introduced in this work. The cytotoxic titer represents the most dilution fold of toxins that results in $50 \%$ loss of cell viability in a dosedependent manner. A cytotoxic titer above ' 50 ' is defined as 'True' output or ' 1 ' (cell dead), and a titer below '50' as 'False' input or ' 0 ' (cell alive). And that comes to be the same towards $\mathrm{Hbl}$ and CytK, as shown in Fig. 1a. Only in the absence of any input $(0,0,0)$, the logic circuit is in the dissociated form with 'False' input (0), and all cells survive. In the presence of any input, it will report as a 'True' output, cells dead. Fig. 1b-d show the basic logic gates involved in the above cellular logic circuit. Both the three components of Nhe and those of $\mathrm{Hbl}$ construct the three-input 'AND' gates (Fig. 1b). The single input of CytK consists of a 'Buffer' gate (Fig. 1c). Meanwhile, the tripartite Nhe or $\mathrm{Hbl}$ can also be regarded as one whole input to build another two 'Buffer' gates, since most B. cereus strains produce the completed sets of Nhe or Hbl. Hence, all Nhe, $\mathrm{Hbl}$ and CytK are utilized as three inputs to construct an 'OR' gate (Fig. 1d). a)

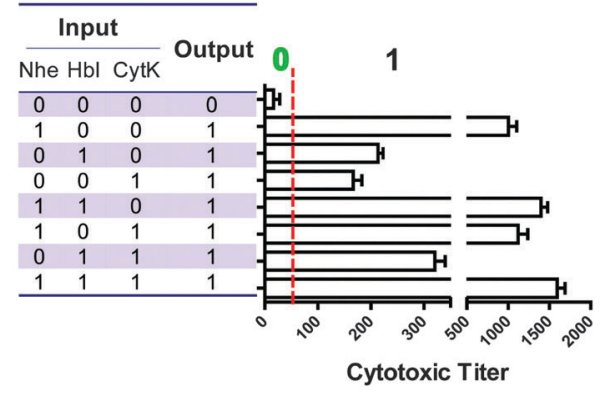

b)

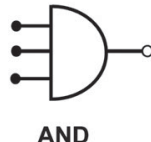

c)

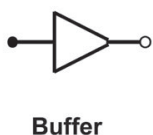

d)

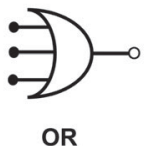

Fig. $1 \mathrm{Nhe}, \mathrm{Hbl}$ and CytK based logic gates. (a) Truth table of the cellular OR gate and the associated cytotoxic titers. The threshold value of the cytotoxic titer (50) is labeled by red dashed line. Equivalent circuit of an AND gate (b), a Buffer gate (c) and an OR gate (d).

To optimize the cellular logic circuit for the detection of toxins from $B$. cereus, we firstly investigated the output signals of Vero cells using three different techniques (Scheme 1a). The cytotoxic titers of Vero cells with the Nhe treatment were detected by LDH, PI and WST assays, respectively, as shown in Fig. 2a. All three methods show similar half maximal inhibitory concentrations $\left(\mathrm{IC}_{50}\right)$ with cytotoxic titers of approximately 1000, which means the lowest concentration of Nhe causing 50\% Vero cells dead. In comparison among the procedures of three assays (schemes of each assay are provided in Fig. S2, ESI $\ddagger$ ), WST assay is the most simplest and easy-to-use
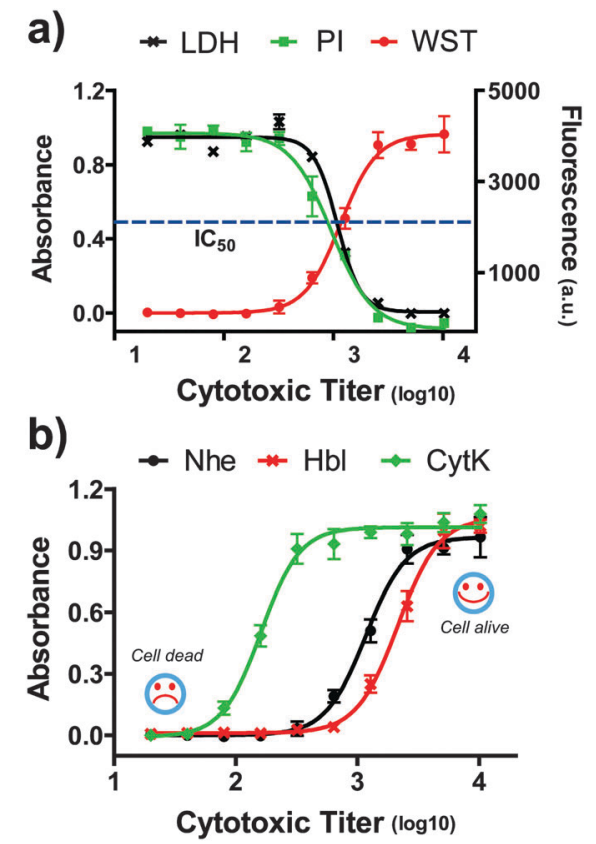

Fig. 2 (a) Comparison of Nhe treated Vero cells using LDH, PI and WST assays. The $I C_{50}$ of Nhe in three assays is labeled by the blue dashed line. (b) Standard curves of the reference strains with $\mathrm{Nhe}, \mathrm{Hbl}$ and CytK production for the cytotoxicity of Vero cells based on WST assays. 
colorimetric method. Because: (1) LDH assay needs not only one more step for centrifugation to obtain the LDH supernatant, but also an extra 96-well microplate to transfer the supernatant; (2) PI assay uses a fluorescent probe which is easily quenched by lights, for binding to cellular DNA and equipped with a specifical 96-well black microplate. Therefore, WST assay is used as a standard method for the following study in this work. Fig. 2b shows the standard curves of Nhe, $\mathrm{Hbl}$ and CytK (from reference strains, details are given in ESI $\ddagger$ ) treated Vero cells in WST assays, the $\mathrm{Hbl}$ positive strain shows the highest toxicity to Vero cells and the CytK strain is the lowest one. And similar results were also obtained when using $\mathrm{LDH}$ and PI assays for the same Nhe, Hbl and CytK toxins (Fig. S3, $\mathrm{ESI} \ddagger)$.

To further confirm the presence of Nhe, $\mathrm{Hbl}$ and CytK expressed by the reference strains, specific primers for each gene were used for PCR detection and ELISAs for the individual proteinic components were performed based on six different monoclonal antibodies (mAbs). All three strains harbor nhe genes, MHI 1505 and MHI 1307 contain additional $h b l$ and cytk genes, respectively, compared with the sole nhe gene in MHI 1491, in Fig. S4 (ESI†). The expressed six protein components of $n h e$ and $h b l$ genes were detected by ELISAs. The presence of NheA, B and C was found both in MHI 1491 and MHI 1505 and Hbl-L1, L2 and B were also positive in MHI 1505 (Fig. S5, ESI $\ddagger$ ). Due to the lack of proper antibody against CytK and the fact that despite some strains harbor $c y t k$ genes without the expression of toxin, this developed cellular logic circuit provides an alternative for this situation.

Robust methods for the analysis of toxin production will not only facilitate the understanding of pathogenesis but also provide useful information for diagnosis and food safety. ${ }^{13}$ Given the huge diversity of PFTs, many approaches have been developed. ${ }^{14}$ These methods can be grouped into two general catalogues: those based on the direct detection of PFT genes or the proteinic components, such as PCR and ELISA; and those based on the indirect determination of activity tests, e.g., the released LDH from the cytosol of PFT treated cells can cause a significant color change in the indicator, as shown in Scheme 1a. For proof-of-concept experiments, the cellular logic circuit was challenged with $20 \mathrm{~B}$. cereus strains derived from food related samples, and the toxin profiles were deciphered using PCR and ELISA as well (Table S1, ESI $\ddagger$ ). All the strains possess nhe genes, but only partial components or none are expressed in some strains such as MHI 1647, MHI 1761 and MHI 1676, which were also confirmed by specific mAbs. Moreover, although the sandwich ELISA for NheB presents a negative result for MHI 2968, it is proved to be a false negative result based on the sophisticated cellular logic circuit which thus shows a higher accuracy than that of ELISA. The reason may be that the mutation in the epitope of NheB recognized by mAbs significantly decreases the affinity for the antibody binding. Both gene and protein based methodologies suffer from certain drawbacks, but the newly developed cellular logic circuit has particular advantages. For example, all the three components of Nhe are needed to trigger full cytotoxicity rather than the presence of $n h e$ genes (PCR positive result) or incomplete expression of one or two components (ELISA positive result).

In conclusion, we have described a logic circuit based on mammalian cells for the rapid detection of toxin production in B. cereus, with simple binary Yes/No answers. The distinctive advantage of the newly proposed assay will certainly accelerate the screening and identification of new pathogenic strains and probiotics compared with the traditional assays based on the presence of genes or single protein components, such as PCR and ELISA. Among numerous abiotic logic gates reported to date, few applications have been achieved to solve challenging practical problems. We have demonstrated herein that the mammalian cellular logic circuit can be used to perform a sophisticated analytical task efficiently and simply with multiple outputs. This work provides a versatile platform for studying the interaction between bacteria and cells integrating Boolean logic operations and biology.

\section{Notes and references}

1 J. Pizarro-Cerdá and P. Cossart, Cell, 2006, 124, 715.

2 M. Bielaszewska, A. Mellmann, W. L. Zhang, R. Köck, A. Fruth, A. Bauwens, G. Peters and H. Karch, Lancet Infect. Dis., 2011, 11, 671.

3 (a) R. Aroian and F. G. van der Goot, Curr. Opin. Microbiol., 2007, 10, 57; (b) R. J. Gilbert, Cell Mol. Life Sci., 2002, 59, 832.

4 (a) M. Bischofberger, I. Iacovache and F. G. van der Goot, Cell Host Microbe, 2012, 12, 266; (b) M. Mueller, U. Grauschopf, T. Maier, R. Glockshuber and N. Ban, Nature, 2009, 459, 726.

5 (a) N. Groulx, H. McGuire, R. Laprade, J. L. Schwartz and R. Blunck, J. Biol. Chem., 2011, 286, 42274; (b) M. Bischofberger, M. R. Gonzalez and F. G. van der Goot, Curr. Opin. Cell Biol., 2009, 21, 589.

6 (a) L. S. Arnesen, A. Fagerlund and P. E. Granum, FEMS Microbiol. Rev., 2008, 32, 579; (b) J. L. Schoeni and A. C. Wong, J. Food Prot., $2005,68,636$.

7 D. J. Beecher and A. C. Wong, J. Biol. Chem., 1997, 272, 233.

8 (a) Z. Ezziane, Nanotechnology, 2006, 17, R27; (b) Bioelectronics: From Theory to Applications, ed. I. Willner and E. Katz, Wiley-VCH, Weinheim, 2005; (c) E. Katz and V. Privman, Chem. Soc. Rev., 2010, 39, 1835.

9 (a) E. Katz, J. Wang, M. Privman and J. Halámek, Anal. Chem., 2012, 84, 5463; (b) E. E. May, P. L. Dolan, P. S. Crozier, S. Brozik and M. Manginell, IEEE Sens. J., 2008, 8, 1011; (c) G. von Maltzahn, T. J. Harris, J. H. Park, D. H. Min, A. J. Schmidt, M. J. Sailor and S. N. Bhatia, J. Am. Chem. Soc., 2007, 129, 6064.

10 (a) M. A. TerAvest, Z. Li and L. T. Angenent, Energy Environ. Sci., 2011, 4, 4907; (b) Z. Li, M. A. Rosenbaum, A. Venkataraman, T. K. Tam, E. Katz and L. T. Angenent, Chem. Commun., 2011, 47, 3060; (c) M. A. Arugula, N. Shroff, E. Katz and Z. He, Chem. Commun., 2012, 48, 10174; (d) J. M. Callura, D. J. Dwyer, F. J. Isaacs, C. R. Cantor and J. J. Collins, Proc. Natl. Acad. Sci. U. S. A., 2010, 107, 15898; (e) T. K. Lu, A. S. Khalil and J. J. Collins, Nat. Biotechnol., 2009, 27, 1139.

11 (a) S. Ausländer, D. Ausländer, M. Müller, M. Wieland and M. Fussenegger, Nature, 2012, 487, 123; (b) Z. Xie, L. Wroblewska, L. Prochazka, R. Weiss and Y. Benenson, Science, 2011, 333, 1307; (c) K. Rinaudo, L. Bleris, R. Maddamsetti, S. Subramanian, R. Weiss and Y. Benenson, Nat. Biotechnol., 2007, 25, 795.

12 (a) L. Halámková, J. Halámek, V. Bocharova, S. Wolf, K. E. Mulier, G. Beilman, J. Wang and E. Katz, Analyst, 2012, 137, 1768; (b) J. Zhou, J. Halámek, V. Bocharova, J. Wang and E. Katz, Talanta, 2011, 83, 955; (c) J. Halámek, V. Bocharova, S. Chinnapareddy, J. R. Windmiller, G. Strack, M. C. Chuang, J. Zhou, P. Santhosh, G. V. Ramirez, M. A. Arugula, J. Wang and E. Katz, Mol. BioSyst., 2010, 6, 2554.

13 (a) O. Lazcka, F. J. D. Campo and F. X. Munoz, Biosens. Bioelectron., 2007, 22, 1205; (b) Y. Shlyapnikov, E. Shlyapnikova, M. Simonova, A. Shepelyakovskaya, F. Brovko, R. Komaleva, E. Grishin and V. Morozov, Anal. Chem., 2012, 84, 5596.

14 V. Havlicek, K. Lemr and K. A. Schug, Anal. Chem., 2013, 85, 790. 